\title{
Semiparametric Multi State Model for Time-To-Event Data
}

\author{
Ramakrishnan .M $\mathbf{M}^{1}$, Viswanathan . $\mathrm{N}^{2}$ \\ ${ }^{1}$ Assistant professor, Department of Mathematics, RKM Vivekananda College, Chennai, India \\ ${ }^{2}$ Assistant Professor, Department of Statistics, Presidency College, Chennai, India
}

\begin{abstract}
Survival Analysis is the study about time-to-event data. It stands apart from classical estimation, in the sense that it has censoring objects with incomplete information to be dealt with. Classical Survival models usually contain two events, of which one is treated as terminal event. Multi State Models (MSM) involve more than two states, of which some may be transient and others absorbing. The multi-state Markov model is a useful way of describing a process in which an individual moves through a series of states in continuous time. Multi-state models can be used to model the movement of patients between different states, such as, hospitalization, recovery, relapse and death. These models may offer a better understanding of the process due to transition specific nature of the events. Also, the estimated transition probabilities from one state to another throws more light on the nature of movements and the possible reasons behind the transitions. In this paper, a multi state model with three states is considered under Semiparametric multi state approach. This method enables us to identify transition specific covariates that throw more light on the entire transition process and the factors influencing the same. A Cox Proportional hazard multi state model is used to derive the necessary estimates and testing procedures are carried out using 'mstate' package of $R$, a open source software.
\end{abstract}

Keywords: Multi State Markov Model, Three State Survival Model, Cox PH model, Transition specific covariates

\section{Introduction}

Survival analysis is the analysis of data measured from a specific time of origin until an event of interest or a specified endpoint (Collett, 1994). For example, in order to determine the incidence of death due to AIDS among HIV positive patients, every patient will be followed from a baseline date (such as date of diagnosis) until the date of death due to AIDS or study closing date. A patient who dies of HIV during the study period would be considered to have an 'event' at the date of death. A patient who is alive at the end of the study would be considered to be 'censored'. Thus, every patient provides two pieces of information: follow-up time and status (censored status). This model is called as Classical Survival model. In longitudinal studies, patients are observed over time and covariate information is collected at several occasions. In such studies, some state may be partitioned into two or more intermediate (transient) states, each of which corresponds to a particular stage.

\section{Multi state Models}

The simplest form of MSM is the mortality model for survival analysis with states "alive" and "dead" and only one possible transition. Splitting the "alive" state into two transient states, it is called as simplest progressive threestate model. Both models are special cases of the kprogressive model, illustrated in Figure 2.1

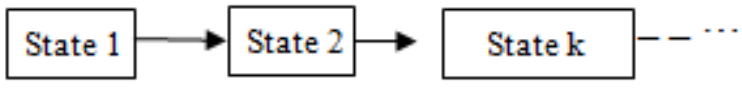

Figure 2.1: k- Progressive Model

Another MSM used for the disease progression is the illnessdeath model which can be used to study the incidence of the disease and the rate of death. This is illustrated in Figure 2.2.

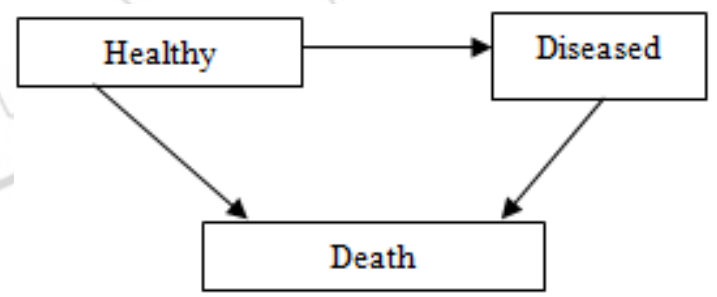

Figure 2.2: Illness-Death Model.

An MSM is a stochastic process $(\mathrm{X}(\mathrm{t}), \mathrm{t} \in \mathrm{T})$ with a finite state space, where $X(t)$ represents the state occupied by the process at time $t \geq 0$. In general, the future state transitions of an MSM may depend on past events. However, for the special case of a Markov model the past and future are independent given its present state. Extensive literature on MSMs are available. Main contribution include books by Anderson, Borgan, Gill, and Keiding (1993), Hougaard (1999), Beyersmann, Schumacher and Allignol (2012) and Willekens, F. (2014). Recent reviews on this topic may be found in the papers by Putter, Fiocco, and Geskus (2007), Putter (2014). The 'mstate' package in R for the analysis of multi-state models was developed by Wreede, Fiocco and Putter (2011).

\subsection{Preliminaries in Multi State Models}

2.2.1Transition intensity If $T$ denotes the time of reaching state $\mathrm{j}$ from state $\mathrm{i}$ then hazard rate (transition intensity) of the $\mathrm{i}$ transition is

$$
\lambda_{i j}(t)=\lim _{\Delta t \rightarrow 0} \frac{\operatorname{Prob}(t \leq T<t+\Delta t \mid T \geq t)}{\Delta t}
$$

This definition makes an assumption that the multi-state model is Markovian, which implies that the probability of going to future state depends only on the present state and not on its history. 


\section{International Journal of Science and Research (IJSR) \\ ISSN (Online): 2319-7064 \\ Index Copernicus Value (2013): 6.14 | Impact Factor (2015): 6.391}

2.2.2 Cumulative transition hazard

Cumulative transition hazard for transition $\mathrm{i}$ j is defined as

$$
\left.\overrightarrow{\Lambda_{i j}} \vec{t}\right)=\int_{0}^{t} \lambda_{i j}(s) d s
$$

\subsubsection{Cox Proportional Hazards model}

The hazard for the transition $\mathrm{i} \rightarrow \mathrm{j}$ for a subject with covariate vector $\mathbf{Z}$ is

$$
\lambda_{i j}(t \mid \boldsymbol{Z})=\lambda_{i j, 0}(t) \exp \left(\boldsymbol{\beta}_{i j}^{T} \boldsymbol{Z}\right)
$$

where $\lambda_{i j, 0}(t)$ is the baseline hazard of transition $\mathrm{i} \rightarrow \mathrm{j}$, and $\beta_{i j}$ is the vector of regression coefficients that describe the effect of $\mathbf{Z}$ on transition $\mathrm{i} \rightarrow \mathrm{j}$.

\subsubsection{Test based on the Schoenfeld Residuals}

Several formal statistical tests have been proposed for assessment of proportionality of hazards. A simulation study by Ng'andu (1997) described and compared several tests in the Cox PH framework, and concluded that the scaled Schoenfeld residuals test (Grambsch and Therneau,1994), the linear correlation test (Harrell, 1986) and the timedependent covariate test(Cox, 1972) were the most powerful diagnostic tools for proportionality. The other statistical test of the proportional hazards assumption is based on the Schoenfeld residual. The Schoenfeld residuals are defined for each subject who is observed to fail. If the $\mathrm{PH}$ assumption holds for a particular covariate then the Schoenfeld residual for that covariate will be independent of survival time. So this test is accomplished by finding the correlation between the Schoenfeld residuals for a particular covariate and the ranking of individual survival times. The null hypothesis is that the correlation between the Schoenfeld residuals and the ranked survival time is zero. Rejection of null hypothesis concludes that $\mathrm{PH}$ assumption is violated.

The scaled residuals scatter in a nonsystematic way around the zero line, and the polygon connecting the values of the smoothed residuals has approximately a zero slope and crosses the zero line several times. Then we conclude that hazard function may be proportional in that covariate. If the polygons connecting the values of smoothed residuals have not zero slope and crosses the zero line only once or it has consistent positive slope, suggesting that the importance of the covariate increases over time and thus has a non proportional hazard.

\subsubsection{Approaches in Time Scale}

Clock forward: Time $t$ refers to the time since the patient entered the initial state.

Clock reset: Time $t$ in $\lambda_{i j}(t)$ refers to the time since entry in state $i$. The clock is reset to 0 each time the patient enters a new state.

\section{Applications to German Breast Cancer Study (GBCS)}

\subsection{Data and Model Description}

GBCS data obtained from the German Breast Cancer Study Group, which they used to illustrate the methods for building

prognostic models (Sauerbrei and Royston, 1999) is used for this study. In the main study, a total of 720 patients with primary node positive breast cancer were recruited between July 1984, and December 1989. Data used in this study consists of 686 subjects with complete data contain three events primary node positive, recurrence and death respectively. Using Purposeful selection of covariates method, the following prognostic factors were identified and their distribution of the values is shown in table 3.1

387(299) 128(171)

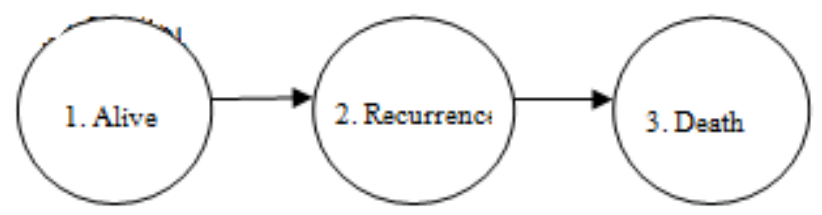

Figure 3.1 Transition numbers of events and Censored observations

(Figures inside the bracket denotes the events)

Table 3.1: Distribution of Prognostic factors

\begin{tabular}{|c|c|c|c|}
\hline Progonastic factor & Categories & Number & in \% \\
\hline Hormone Therapy & No & 440 & 64 \\
\hline & Yes & 246 & 36 \\
\hline Tumour Grade & 1 & 81 & 12 \\
\hline & 2 & 444 & 65 \\
\hline Number of Nodes & 3 & 161 & 23 \\
\hline Age & $<4$ & 376 & 55 \\
\hline & $\geq 4$ & 310 & 45 \\
\hline & $(20,46]$ & 181 & 26 \\
\hline & $(46,53]$ & 182 & 27 \\
\hline & $(53,61]$ & 155 & 23 \\
\hline Numour Size in mm & $(61,81]$ & 168 & 24 \\
\hline & $(2,25]$ & 353 & 51 \\
\hline & $(25,120]$ & 333 & 49 \\
\hline & $<33$ & 343 & 50 \\
\hline & $\geq 33$ & 343 & 50 \\
\hline
\end{tabular}

The above table indicates that the covariate values are evenly spread among different categories.

The model development and testing are carried out using 'mstate' package in $\mathrm{R}$ and the corresponding outputs are provided below:

Table 3.2: Parameter estimates using 'Clock forward' approach

\begin{tabular}{|c|c|c|c|c|c|}
\hline covariates & coef & exp(coef) & $\mathrm{SE}$ (coef) & $\mathrm{z}$ & $\mathrm{p}$ \\
\hline hormone. 1 & -0.4241 & 0.6543 & 0.1283 & -3.3100 & 0.0010 \\
\hline hormone. 2 & -0.1633 & 0.8493 & 0.1882 & -0.8700 & 0.3856 \\
\hline grade.1 & 0.1686 & 1.1836 & 0.1106 & 1.5200 & 0.1274 \\
\hline grade. 2 & 0.1536 & 1.1660 & 0.1691 & 0.9100 & 0.3639 \\
\hline nodescut.1 & 0.9228 & 2.5163 & 0.1219 & 7.5700 & 0.0000 \\
\hline nodescut.2 & 0.2311 & 1.2600 & 0.1901 & 1.2200 & 0.2241 \\
\hline agecut1.1 & -0.1555 & 0.8560 & 0.1649 & -0.9400 & 0.3458 \\
\hline agecut1.2 & 0.5653 & 1.7600 & 0.2263 & 2.5000 & 0.0125 \\
\hline agecut2.1 & 0.1718 & 1.1874 & 0.1632 & 1.0500 & 0.2926 \\
\hline agecut2.2 & 0.3503 & 1.4195 & 0.2396 & 1.4600 & 0.1437 \\
\hline agecut3.1 & 0.0530 & 1.0544 & 0.1628 & 0.3300 & 0.7449 \\
\hline agecut3.2 & 0.4018 & 1.4945 & 0.2379 & 1.6900 & 0.0913 \\
\hline sizecut.1 & 0.0895 & 1.0936 & 0.1210 & 0.7400 & 0.4593 \\
\hline sizecut.2 & 0.2905 & 1.3372 & 0.1730 & 1.6800 & 0.0931 \\
\hline progcut.1 & -0.6552 & 0.5194 & 0.1276 & -5.1400 & 0.0000 \\
\hline progcut.2 & -0.8421 & 0.4308 & 0.2122 & -3.9700 & 0.0001 \\
\hline
\end{tabular}

Volume 5 Issue 5, May 2016 www.ijsr.net 


\section{International Journal of Science and Research (IJSR) ISSN (Online): 2319-7064 \\ Index Copernicus Value (2013): 6.14 | Impact Factor (2015): 6.391}

Table 3.3: Test of the proportional hazards assumption based on the Schoenfeld residual using 'Clock forward' approach

\begin{tabular}{|c|c|c|c|}
\hline covariates & rho & Chi-square & $\mathrm{p}$ \\
\hline hormone. 1 & -0.0116 & 0.0607 & 0.8054 \\
\hline hormone. 2 & 0.0442 & 0.9429 & 0.3315 \\
\hline grade.1 & -0.0864 & 2.9429 & 0.0863 \\
\hline grade. 2 & -0.0235 & 0.2602 & 0.6100 \\
\hline nodescut.1 & -0.0838 & 3.0185 & 0.0823 \\
\hline nodescut.2 & 0.0142 & 0.1079 & 0.7426 \\
\hline agecut1.1 & 0.0440 & 0.8661 & 0.3520 \\
\hline agecut1.2 & 0.0214 & 0.2144 & 0.6433 \\
\hline agecut2.1 & 0.1270 & 7.1586 & 0.0075 \\
\hline agecut2.2 & 0.0170 & 0.1243 & 0.7244 \\
\hline agecut3.1 & 0.1009 & 4.5632 & 0.0327 \\
\hline agecut3.2 & -0.0588 & 1.5368 & 0.2151 \\
\hline sizecut.1 & 0.0086 & 0.0347 & 0.8523 \\
\hline sizecut.2 & -0.0024 & 0.0027 & 0.9588 \\
\hline progcut.1 & 0.1420 & 8.8152 & 0.0030 \\
\hline progcut.2 & 0.0873 & 3.6615 & 0.0557 \\
\hline GLOBAL & $\mathrm{NA}$ & 38.3936 & 0.0013 \\
\hline & & &
\end{tabular}

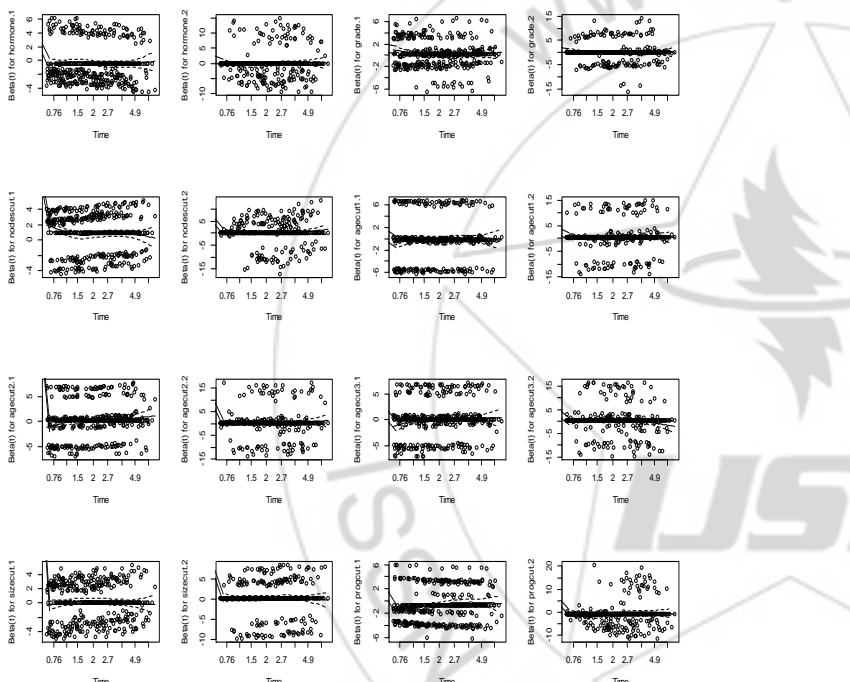

Figure 3.2: Scatter plot of scaled Schoenfeld residuals using 'Clock forward' approach

Table 3.4: Parameter estimates using 'Clock reset' approach

\begin{tabular}{|c|c|c|c|c|c|}
\hline Parameters & coef & $\exp ($ coef $)$ & $\mathrm{se}($ coef $)$ & $\mathrm{z}$ & $\mathrm{p}$ \\
\hline hormone.1 & -0.4241 & 0.6543 & 0.1283 & -3.3100 & $0.0009500^{*}$ \\
\hline hormone.2 & -0.1536 & 0.8576 & 0.1713 & -0.9000 & 0.3698 \\
\hline grade.1 & 0.1686 & 1.1836 & 0.1106 & 1.5200 & 0.1274 \\
\hline grade.2 & 0.1925 & 1.2122 & 0.1573 & 1.2200 & 0.2212 \\
\hline nodescut.1 & 0.9228 & 2.5163 & 0.1219 & 7.5700 & $0.0000000^{*}$ \\
\hline nodescut.2 & 0.2833 & 1.3275 & 0.1721 & 1.6500 & 0.0998 \\
\hline agecut1.1 & -0.1555 & 0.8560 & 0.1649 & -0.9400 & 0.3458 \\
\hline agecut1.2 & 0.6289 & 1.8756 & 0.2167 & 2.9000 & $0.0037100^{*}$ \\
\hline agecut2.1 & 0.1718 & 1.1874 & 0.1632 & 1.0500 & 0.2926 \\
\hline agecut2.2 & 0.4134 & 1.5120 & 0.2290 & 1.8100 & 0.0710 \\
\hline agecut3.1 & 0.0530 & 1.0544 & 0.1628 & 0.3300 & 0.7449 \\
\hline agecut3.2 & 0.4912 & 1.6342 & 0.2228 & 2.2000 & $0.0275100^{*}$ \\
\hline sizecut.1 & 0.0895 & 1.0936 & 0.1210 & 0.7400 & 0.4593 \\
\hline sizecut.2 & 0.2148 & 1.2396 & 0.1616 & 1.3300 & 0.1838 \\
\hline progcut.1 & -0.6552 & 0.5194 & 0.1276 & -5.1400 & $0.0000003^{*}$ \\
\hline progcut.2 & -0.8387 & 0.4323 & 0.1945 & -4.3100 & $0.0000160^{*}$ \\
\hline
\end{tabular}

*Denotes significance at $5 \%$ level.
Table 3.5: Test of the proportional hazards assumption based on the Schoenfeld residual using 'Clock reset' approach

\begin{tabular}{|c|c|c|c|}
\hline Parameters & rho & chi-square & $\mathrm{p}$ \\
\hline hormone. 1 & -0.0108 & 0.0549 & 0.8147 \\
\hline hormone. 2 & -0.0048 & 0.0117 & 0.9137 \\
\hline grade.1 & -0.0748 & 2.3100 & 0.1287 \\
\hline grade. 2 & -0.0101 & 0.0507 & 0.8219 \\
\hline nodescut.1 & -0.0757 & 2.5800 & 0.1082 \\
\hline nodescut.2 & 0.0302 & 0.4950 & 0.4818 \\
\hline agecut1.1 & 0.0386 & 0.7000 & 0.4028 \\
\hline agecut1.2 & -0.0366 & 0.6280 & 0.4281 \\
\hline agecut2.1 & 0.1110 & 5.7300 & $0.01670^{*}$ \\
\hline agecut2.2 & -0.0153 & 0.1070 & 0.7435 \\
\hline agecut3.1 & 0.0882 & 3.6500 & 0.0562 \\
\hline agecut3.2 & -0.0975 & 4.3400 & 0.0373 \\
\hline sizecut.1 & 0.0092 & 0.0412 & 0.8392 \\
\hline sizecut.2 & -0.0001 & 0.0000 & 0.9990 \\
\hline progcut.1 & 0.1200 & 6.5900 & $0.01020^{*}$ \\
\hline progcut.2 & 0.0169 & 0.1500 & 0.6984 \\
\hline GLOBAL & $\mathrm{NA}$ & 29.6000 & 0.0204 \\
\hline
\end{tabular}

*Denotes significance at $5 \%$ level.

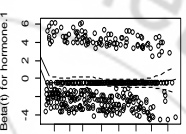
$\begin{array}{lllllll}1 & 1 & 1 & 1 & 1 & 1 \\ 0.76 & 1.5 & 2 & 2.7 & 4.9\end{array}$

$$
\text { Time }
$$

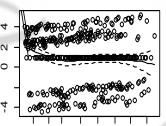

$\begin{array}{llllll}1 & 1 & 1 & 1 & 1 & 1 \\ 076 & 15 & 2 & 27 & 49\end{array}$

$\mathrm{Tr}$

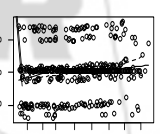
$\begin{array}{lllll}0.5 & 0\end{array}$ Tme

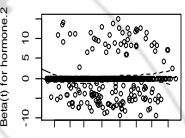

$\begin{array}{llllll}0.76 & 1.5 & 2 & 2 & 2.7 & 4.9\end{array}$

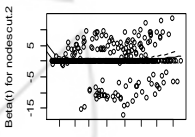

Time
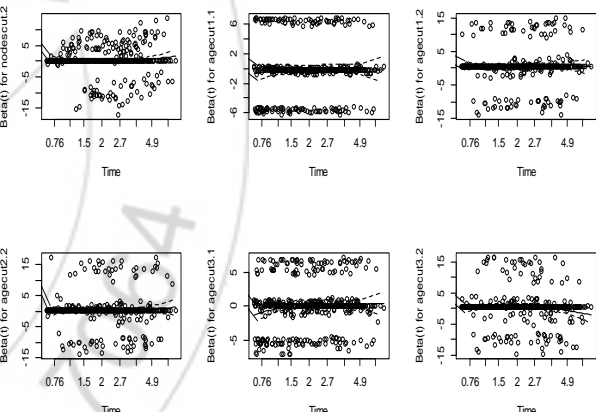

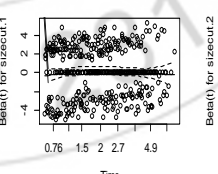

Time

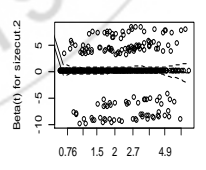

Time

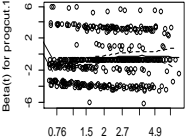

$\begin{array}{llll}176 & 1.5 & 2 & 2.7\end{array}$

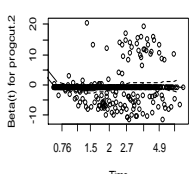

Time
Figure 3.3: Scatter plot of scaled Schoenfeld residuals using 'Clock reset' approach

\section{Summary and Conclusion}

Multi state model identifies significance of each state and their respective transitions in survival analysis. In this study, subjects who have undergone hormone therapy have recurrence at a rate $35 \%$ lower than subjects who do not undergo hormone therapy. In transition 1, subjects who have more than three tumor nodes have higher hazard than those with lesser number of nodes. In both transitions, subjects having higher (greater than 33) number of Progesterone receptors have lower hazard compared to those with relatively lower number of Progesterone receptors. Difference between 'clock forward' and 'clock reset' approaches is quite small with regard to the estimated 


\section{International Journal of Science and Research (IJSR) \\ ISSN (Online): 2319-7064}

Index Copernicus Value (2013): 6.14 | Impact Factor (2015): 6.391

regression coefficients. No evidence of non-proportionality of the base line transition intensities are seen in the two transitions ( $\mathrm{P}$ value $>0.05$ ), except for a few subclasses in the covariates. Multistate model, when compared to classical survival models, brings out extra features that aid in analyzing survival structures.

\section{References}

[1] Aalen, O.O. (1978). Non parametric inference for afamily of counting processes. Annals of Statistics, 6, 701-726.

[2] Kaplan, E.L. and Meier, P. (1958). Nonparametric estimation from incomplete observations. Journal of American Statistical Association, 53, 457-481.

[3] Meira-Machado L, Cadarso Suarez C and Una-Alvarez J. (2008). Inference in the progressive three-state model. International journal of Mathematical Models and Methods in Applied Scicnces,2(3), 447-454.

[4] Meira-Machado L, Cadarso Suarez C, Una-Alvarez J and Andersen, K.(2009). Multi-state models for the analysis of time-to-event data. Statistical Methods Medical Research,18(2), 195-222.

[5] Nelson.W. (1972). Theory and Applications of Hazard Plotting for Censored Failure Data. Technometrics, 14, 945-966.

[6] Putter, H, Fiocco, M and Geskus, R.B. (2006). Tutorial in biostatistics: Competing risks and multi-state models. Statistics in Medicine,26, 2389-2430.

[7] Sadagopan, JM, Ben-Porat, L, Berwick, M, Robson, M, Kutler, D and Auerbach, AD. (2004). A note on competing risks in survival data analysis. British Journal of Cancer, 91, 1229-1235.

[8] Willekens, F. (2014). Multi state Analysis of Life Histories with R. New York: Springer series.

[9] Wreede,L.C, Fiocco, M and Putter, H. (2011). mstate:An R Package for the Analysis of Competing Risks and Multi-State Models. Journal of Statistical Software, 38(7), 1-30.

\section{Author Profile}
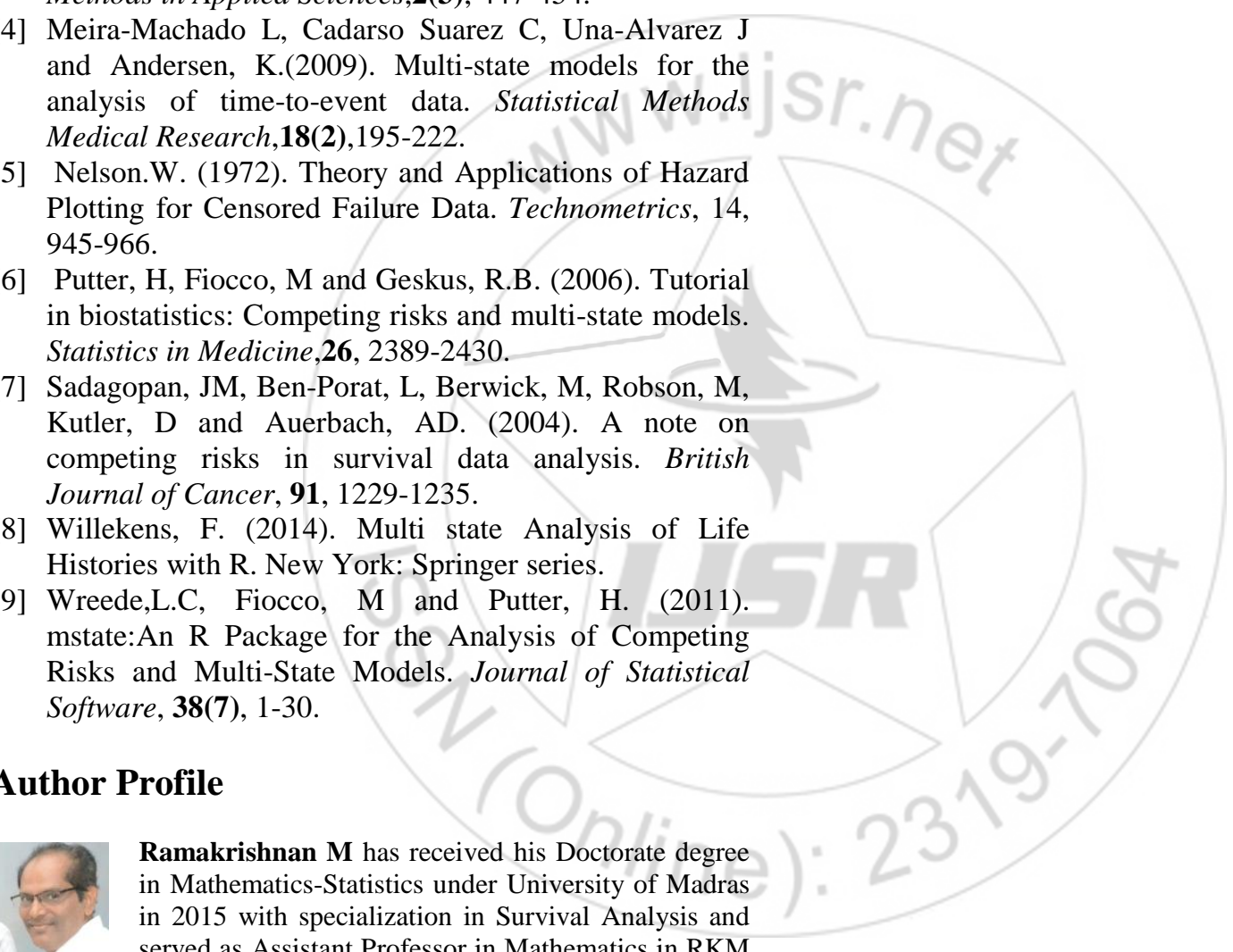

Ramakrishnan M has received his Doctorate degree in Mathematics-Statistics under University of Madras in 2015 with specialization in Survival Analysis and served as Assistant Professor in Mathematics in RKM

Vivekananda College, Chennai from 2008 to 2016.

Viswanathan. $\mathbf{N}$ has received his Doctorate degree in Statistics under University of Madras in 2016 with specialization in Survival Analysis and served as Assistant Professor in Statistics in Presidency College, Chennai from 2001 to 2016. 